\title{
Utilidad de la resonancia magnética dinámica para la evaluación de la inestabilidad disco-vertebral vs. la resonancia magnética estática
}

\author{
Utility of the dynamic magnetic resonance for the evaluation of disco-vertebral instability \\ vs. static magnetic resonance
}

\begin{abstract}
Jorge Paz-Gutiérrez ${ }^{1 *}$, Sandra I. Zaragoza-Solís , Ana S. Sánchez-Gómez², Gabriel V. Ortiz-García Arelhi González-Cisneros ${ }^{4}$ y José Jiménez-Avila ${ }^{5}$

${ }^{1}$ Clínica de columna, Centro Médico Puerta de Hierro, Zapopan; ${ }^{2}$ Clínica de columna, Hospital San Javier, Guadalajara; ${ }^{3}$ Clínica de columna, Centro Médico Puerta de Hierro, Zapopan; 'Escuela de Medicina y Ciencias de la Salud, Tecnológico de Monterrey, Campus Guadalajara, Zapopan; ${ }^{5}$ Clínica de columna, Centro Médico Nacional de Occidente, Instituto Mexicano del Seguro Social, Guadalajara. Jalisco, México
\end{abstract}

\section{Resumen}

Introducción: La resonancia magnética dinámica (RMd) es útil para evaluar la columna cervical inestable; evalúa componentes blandos de la columna cervical y movimientos dinámicos cervicales. Objetivo: Describir y analizar la frecuencia de presentación de hernias discales con resonancia magnética estática (RMe) y comparar con la RMd. Método: De diciembre de 2014 a febrero de 2016 se evaluaron pacientes con cervicalgia y sospecha de inestabilidad cervical. Se realizó un estudio observacional, descriptivo y transversal, en el que se analizaron imágenes obtenidas en flexión y extensión mediante un dispositivo compatible con la RM. Resultados: 29 pacientes ingresaron al protocolo y 7 se excluyeron. Promedio de edad: 50 años. De los 22 pacientes, se evaluaron 154 discos intervertebrales, demostrando 52 hernias discales, y el 78\% fueron protrusiones discales. Nivel más afectado: C5 (31\%). Durante las maniobras dinámicas se demostró que 30 fueron inestables, mostrando modificación durante las maniobras de flexión y extensión; 22 hernias se mantuvieron estables. Cinco pacientes demostraron inestabilidad ósea de cuerpos vertebrales. Conclusiones: La RMd puede utilizarse en nuestro medio, ya que el costo de adquisición es bajo y provee información para la evaluación de la inestabilidad del disco vertebral al mostrar hallazgos no visibles en la RMe. Se obtiene un riesgo relativo de 16 y una $p<0.05$.

PALABRAS CLAVE: Resonancia magnética dinámica. Resonancia magnética estática. Inestabilidad disco vertebral.

\begin{abstract}
Introduction: Dynamic magnetic resonance imaging (dMR) is useful to evaluate the unstable cervical spine; it evaluates soft components of the cervical spine and dynamic cervical movements. Objective: To describe and analyze the frequency of presentation of herniated discs with static MRI and compare with dMR. Method: During December 2014 to February 2016 , patients with cervicalgia and suspected cervical instability were evaluated. An observational, descriptive, cross-sectional study was performed, where images obtained in flexion and extension were analyzed using a device compatible with MRI. Results: 29 patients entered the protocol, 7 were excluded. Average age: 50 years. Of the 22 patients, 154 intervertebral discs were evaluated, demonstrating 52 herniated discs, 78\% were disc protrusions. Most affected level: C5 (31\%). During the dynamic maneuvers it was shown that 30 were unstable, showing modification during the maneuvers of flexion and extension, 22 hernias remained stable. Five patients demonstrated bone instability of vertebral bodies. Conclusions: The dMR can be used in our environment since the acquisition cost is low and provides information for the evaluation of spinal disc instability showing findings not visible in sMR. Obtaining a relative risk of 16 and $p<0.05$
\end{abstract}

KEY WORDS: Dynamic magnetic resonance. Static magnetic resonance. Vertebral disc instability.

\section{Correspondencia:}

*Jorge Paz-Gutiérrez

Av. Empresarios, 150, Puerta de Hierro Fecha de recepción: 19-04-2018

C.P. 45116, Zapopan, Jal., México $\quad$ Fecha de aceptación: 11-07-2018

E-mail: josemajimeneza@ hotmail.com

DOI: $10.24875 / C I R U .18000388$

Cir Cir. 2019;87:28-33

Contents available at PubMed www.cirugiaycirujanos.com 


\section{Introducción}

La cervicalgia crónica es una de las principales patologías en la consulta diaria. Esto es cada vez más común, debido a la modificación en los hábitos higiénico-dietéticos, de los cuales los principales son la postura en el ambiente laboral, los deportes con impacto directo en la columna, los antecedentes traumáticos (en especial con efecto latigazo), el sobrepeso y las labores cotidianas que condicionan microtrauma sobre las estructuras de la columna cervical'.

En pacientes con dolor cervical de tipo crónico que muestren alteraciones neurológicas en la exploración física, la resonancia magnética (RM) es un método con una exactitud de aproximadamente el $80 \%$ para el diagnóstico de la anomalía causante ${ }^{2}$.

La resonancia magnética estática (RMe) ha tenido algunas limitaciones en la demostración de la causa de los síntomas dolorosos, ya sea radiculopatía o mielopatía, ya que en una posición en decúbito supino la fuerza gravitacional que se ejerce sobre la columna actuará directamente sobre la cara ventral de los cuerpos vertebrales, permitiendo un aumento en la apertura de los espacios intervertebrales y en ocasiones rectificación de la lordosis cervical, ocultando patologías discales tales como herniaciones, espondilolistesis 0 inestabiliad del cuerpo vertebral. Sin embargo, en una posición en flexión, la fuerza gravitacional se modifica, incrementándose el peso y la fuerza gravitacional en sentido axial sobre la columna cervical, lo que favorece un aumento en la presión intradiscal, simulando la posición en la cual el paciente manifiesta los síntomas y siendo más evidentes las anomalías discales ${ }^{3}$.

La investigación en estos métodos está en evolución, y a pesar de que existen dispositivos en el mercado que favorecen los movimientos dinámicos de la columna vertebral, no existen protocolos que estén aceptados por organizaciones internacionales y en nuestro medio no se cuenta con los tipos de RM necesarios para poder realizar una evaluación dinámica, por lo que muchos pacientes continúan con diagnósticos fallidos ${ }^{4-6}$.

\section{Métodos de imagen}

La enfermedad degenerativa de la columna es compleja y se manifiesta clínicamente como dolor; sin embargo, por imagen podemos encontrar diversos hallazgos que, dependiendo de su evolución, pueden ser más o menos evidentes. La RM es una técnica basada en la excitación de las moléculas de hidrógeno de las células que permite una caracterización volumétrica de las estructuras que conforman la columna cervical, principalmente las estructuras con alto contenido en moléculas de hidrógeno, como lo son los discos intervertebrales, los ligamentos, los músculos y también las estructuras óseas, aunque con menor sensibilidad. Las secuencias Spin Echo nos han permitido identificar la causa de la cervicalgia hasta en un $80 \%$ de los casos.

La nitidez de las estructuras que evaluaremos depende de las Teslas del resonador: a más Teslas, mayor será la nitidez de las imágenes obtenidas. Existen también otros factores que intervienen, como son los relacionados con el paciente (hábito corporal, movimientos voluntarios e involuntarios, presencia de material quirúrgico en la columna o en las estructuras que condicionen ausencia de la señal, malformaciones, etc.), los cuales pueden hacer que las imágenes no sean óptimas para su adecuada evaluación.

La RMe permite la valoración morfológica de las estructuras, pero la resonancia magnética dinámica (RMd) nos permite valorar la alineación de la columna cervical, la cual se verá afectada cuando existan alteraciones en las estructuras que dan estabilidad al eje axial de la columna cervical.

La evolución de la enfermedad degenerativa radiológicamente puede iniciar con rectificación de la columna debido a la contractura de los músculos, y más tarde con formación de osteofitos debido a la tracción de las fibras de Sharpey o por la fuerza tensil condicionada por el ligamento longitudinal anterior sobre el periostio del cuerpo vertebral, con disminución en el espacio intervertebral, lo cual a su vez condiciona cambios en la presión del disco intervetebral con formación de gas intradiscal, así como disminución en el espacio intervetrebral con esclerosis o edema de las facetas subyacentes y suprayacentes, y ya en estadios avanzados fusión de los cuerpos vertebrales.

En una fase intermedia, la formación de los osteofitos pueden presentarse en la cara anterior, posterior o lateral de los cuerpos vertebrales, así como con extensión a los forámenes de conjunción, mismos que a su vez pueden condicionar radiculopatía, siendo esta una de las principales causas de consulta por cervicalgia. De igual manera, en algunos pacientes puede verse calcificación del ligamento amarillo y del ligamento longitudinal, el cual a su vez puede condicionar un canal vertebral estrecho, aunque cabe mencionar que la RM tiene pobre sensibilidad para la evaluación de este hallazgo ${ }^{7}$.

El disco intervetebral, por su alto contenido en líquido, se evalúa mejor con secuencias sensibles al líquido, principalmente T2 o T2 con supresión grasa. Las estructuras óseas se valoran con mayor limitación en 
la RM, pero las secuencias potenciadas en $\mathrm{T} 1$ y T2 son útiles para la visualización de osteofitos. Para la determinacion de cambios de tipo Modic son útiles las secuencias ponderadas en T1, T2 y T2 con supresión de grasa, las cuales determinarán si son cambios por sustitución grasa, edema o esclerosis. Las secuencias T2 en sagital son óptimas para hacer una RMd, ya que esta permitirá una adecuada valoración de los bordes óseos y determinar un desplazamiento anterior o posterior, lo cual estará evaluando la laxitud de los ligamentos que mantienen la estabilidad cervical ${ }^{8-10}$.

Es sumamente importante el uso de métodos de imagen para determinar el grado de inestabilidad y el tratamiento que se llevará a cabo, ya que según los hallazgos se evaluará el requerimiento de cirugía o solo de terapia farmacológica y rehabilitación?.

El diámetro anteroposterior normal del canal medular es evaluado por este método, el cual debe de ser de $22 \mathrm{~mm}$ a nivel de $\mathrm{C} 2$ y de $18 \mathrm{~mm}$ de $\mathrm{C} 3$ a C6, diagnosticándose un canal estrecho cuando es inferior a $14 \mathrm{~mm}$ y con clínica significativa cuando es menor de $11 \mathrm{~mm}$; de igual manera, este puede verse reducido en los movimientos dinámicos ${ }^{11-13}$.

Se ha reportado que las secuencias rápidas Spin Echo suelen tener una sensibilidad de más del $80 \%$ para evaluar los forámenes y el canal vertebral. Actualmente no se ha demostrado un beneficio adicional con el uso de medio de contraste agregado, por lo que en general las RM utilizadas en la enfermedad degenerativa suelen ser simples ${ }^{14,15}$.

\section{Tratamiento}

El objetivo es fijar el segmento inestable, que si es exitoso y sólido se vuelve asintomático. La segunda alternativa es restablecer la estabilidad, pero mantener la movilidad del segmento mediante estabilización flexible con el uso de prótesis o cajas intersomáticas. En tercer lugar, se han promovido varias prótesis de reemplazo de disco para restaurar las propiedades estructurales y biomecánicas necesarias de la columna vertebral que se necesitan para conseguir la lordosis fisiológica y la estabilidad de los segmentos móviles ${ }^{16}$.

Las indicaciones de un tratamiento quirúrgico son principalmente la determinación de una columna cervical inestable. Actualmente, con lo único que contamos es con los criterios de White y Panjabi ${ }^{15}$, que se basan en hacer una suma de los hallazgos clínico-radiológicos de inestabilidad y, si se encuentran más de 5 puntos, se determinará una columna inestable (Tabla 1).
Tabla 1. Lista de verificación para el diagnóstico de inestabilidad clínica en la columna cervical media e inferior.

Elemento
Elementos anteriores destruidos o incapaces de funcionar
Elementos posteriores destruidos o incapaces de funcionar
Prueba de estiramiento positivo
Criterios radiográficos
A. Rayos $X$ de flexión-extensión
1. Traslado del plano sagital>3.5 $\mathrm{mm}$ o $20 \%$
( 2 puntos)
2. Rotación del plano sagital> $20^{\circ}$ ( 2 puntos)
B. Radiografías de reposo
1. Desplazamiento del plano sagital>3.5 $\mathrm{mm}$ o $20 \%$
(2 puntos)
2. Angulación relativa del plano sagital $>11^{\circ}$
(2 puntos)

Canal medular estrecho en el desarrollo

(diámetro sagital $<13 \mathrm{~mm}$ o relación de Pavlov<0.8)

Reducción anormal del disco

Daño de la médula espinal

Daño a raíz nerviosa

Carga peligrosa prevista

Total de 5 o más=inestable.

Adaptada de White AA, Panjabi MM. Clinical biomechanics of the spine. $2^{\text {nd }} \mathrm{ed}$. Philadelphia: Lippincott; 1990.

Los pacientes tratados con fusión e instrumentación y con descompresión posterior parecen tener el mejor resultado.

Los estudios de imagen tienen un importante papel que ayuda a justificar un tratamiento quirúrgico, ya que son el único método capaz de determinar las anormalidades en la dinámica de los movimientos de la columna. Es por eso que resulta sumamente importante una adecuada valoración imagenológica con métodos que ayuden a determinar la existencia o no de cambios osteodegenerativos que puedan ser corregidos mediante la cirugía o mediante tratamientos no quirúrgicos, y que ayuden a una mejoría en la calidad de vida de los pacientes ${ }^{17,18}$.

\section{Método}

Estudio de cohorte en el que se evaluaron 135 pacientes que acudieron al servicio de radiología e imagen para realizarse una RM de columna con cervicalgia y sospecha de patología discal, espondilolistesis o mielopatía, entre diciembre de 2014 y febrero de 2016, de los cuales 29 tenían el complemento con RMd prescrito por el médico tratante y solo 22 fueron ingresados al protocolo cumpliendo con los criterios de inclusión. 
Se realizó el complemento con RMd, previa solicitud del médico tratante, quien realizó una exploración física y neurológica completa considerando apto al paciente para la realización de movimientos dinámicos de flexión y extensión de la columna cervical. Las imágenes obtenidas fueron evaluadas por un grupo médico multidisciplinario con especialistas en cirugía de columna y en radiología, y con un médico subespecialista en neurorradiología.

Se utilizó un resonador magnético marca Siemens de 3.0 T, Modelo Verio, con apertura del túnel de $70 \mathrm{~cm}$. En todos los pacientes se utilizó una antena para columna cervical de 18 canales con adquisición en paralelo. Se utilizó una estación de trabajo marca Siemens tipo Leonardo MR para la evaluación de las imágenes obtenidas. En todos los pacientes se realizó un protocolo establecido, obteniendo secuencias multiplanares T1 y T2 en los planos axial y sagital, y STIR en sagital, todas ellas en posición en decúbito supino estática, y posteriormente se realizó el complemento dinámico con secuencias T2 en sagital y parasagital, derecha e izquierda, a nivel de los forámenes de conjunción correspondientes para evaluar su obliteración durante los movimientos de flexión y extensión. Los movimientos dinámicos se obtuvieron utilizando un dispositivo con gradación a $-10^{\circ}, 0^{\circ}, 10^{\circ}, 20^{\circ}, 30^{\circ}$ y $40^{\circ}$, con un campo de visión de $25 \times 25 \mathrm{~cm}$ en el que se observó la columna cervical desde la unión atlantodontoidea hasta la unión cervicotorácica con un Tiempo de Recuperación (TR) de 3490 ms y un Tiempo de Echo (TE) de $84 \mathrm{~ms}$, con una totalidad del estudio en un tiempo promedio de 25 minutos. Posteriormente se realizó la evaluación estadística utilizando el programa Epi Info (versión 7.2), permitiéndonos realizar una tabla de $2 \times 2$, para luego calcular el riesgo relativo y el valor de $\mathrm{p}$ y determinar la utilidad de la RMd.

Durante los meses de noviembre y diciembre de 2014 se diseñó un dispositivo elaborado con madera, compatible con el magnetismo del resonador, cubierto por tela vinílica de color negro, que tiene una base rígida móvil, con la capacidad de realizar movimientos de flexión cada $10^{\circ}$ sin necesidad de movimientos activos por parte de los pacientes; para evaluar la hiperextensión a $-10^{\circ}$ se retiraron el dispositivo y la base de la antena, lo que permitió realizar una hiperextensión a dicha gradación.

\section{Resultados}

Se estudiaron 29 pacientes, de los cuales se excluyeron siete $(24 \%)$ debido a que presentaban criterios de no inclusión: cuatro con antecedentes quirúrgicos de instrumentación, dos con lesiones desmielinizantes y uno con diagnóstico de astrocitoma medular. De los pacientes estudiados, $15(68 \%)$ eran mujeres y $7(32 \%)$ eran hombres, con una media de edad de 50 años, con un máximo de 66 y un mínimo de $22(+16$ y -28 años). Todos ellos presentaban clínica de cervicalgia crónica, es decir, con más de 6 meses de dolor cervical o que no mejoraba con el tratamiento habitual. En los 22 pacientes que mostraron hernias discales se evaluaron cada uno de los niveles, en total 154 discos intervertebrales, y se demostraron 52 hernias discales, siendo más común la presencia de protrusiones discales, en un $78 \%$ (120). El nivel más afectado fue $\mathrm{C} 5$, con un $31 \%$ (48). Durante las maniobras dinámicas se demostró que $30(20 \%)$ de ellas fueron inestables, mostrando modificación durante las maniobras de flexión y extensión, y 22 hernias se mantuvieron estables. Otro de los hallazgos significativos fue la presencia de desplazamiento anterior 0 posterior de algún cuerpo vertebral (inestabilidad), la cual se presentó en $5(3 \%)$ pacientes, hallazgo que no había sido evidente en las imágenes de RMe (Figs. 1 a 3).

\section{Conclusiones}

La RMe realizada comúnmente en los hospitales se desarrolla en una posición de decúbito supino, lo que permite que la fuerza gravitacional sea directamente sobre la cara ventral de los cuerpos vertebrales y los discos intervertebrales, condición que favorece una mayor amplitud en los espacios intervertebrales y por lo tanto puede perderse una adecuada visualización de las hernias discales. Otra de las principales diferencias que existen entre la RMe y la RMd es que en esta última se puede evaluar en movimientos de flexión y extensión con diferentes grados de inclinación, para así poder demostrar en qué situaciones va a ser evidente una anomalía morfológica que pueda explicar los síntomas del paciente que no habían sido detectados con ningún otro método.

Una gran ventaja de la RMd es que con los movimientos dinámicos se evalúan no solo los cuerpos vertebrales, sino también la estabilidad de las facetas articulares, los ligamentos, el diámetro del espacio subaracnoideo y el contacto con el cordón medular o su extensión hacia los forámenes de conjunción, ayudando a determinar la causa del dolor.

Los cambios degenerativos iniciales se empiezan a presentar en el disco intervertebral, con rotura gradual 

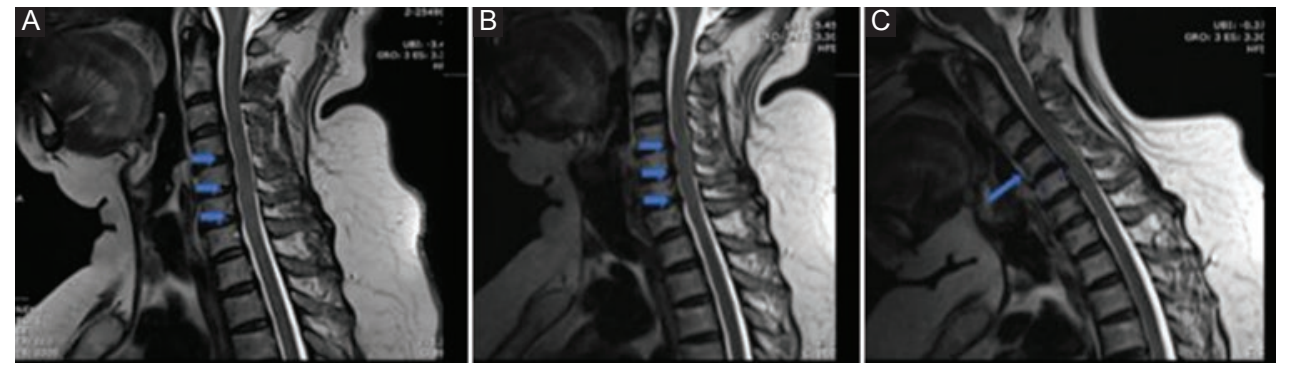

Figura 1. Mujer de 59 años con cervicalgia. A: resonancia magnética (RM) estática con abombamientos discales C4-C5, C5-C6, C6-C7 (flechas). B: RM dinámica con hiperextensión a $-10^{\circ} q u e$ muestra acentuación de las hernias discales con importante disminución del espacio subdural y contacto con el cordón medular. C: flexión a $40^{\circ}$ con desplazamiento anterior de C4 sobre C5; el contacto medular es más evidente (flechas) y la paciente refiere haberse acentuado el dolor.
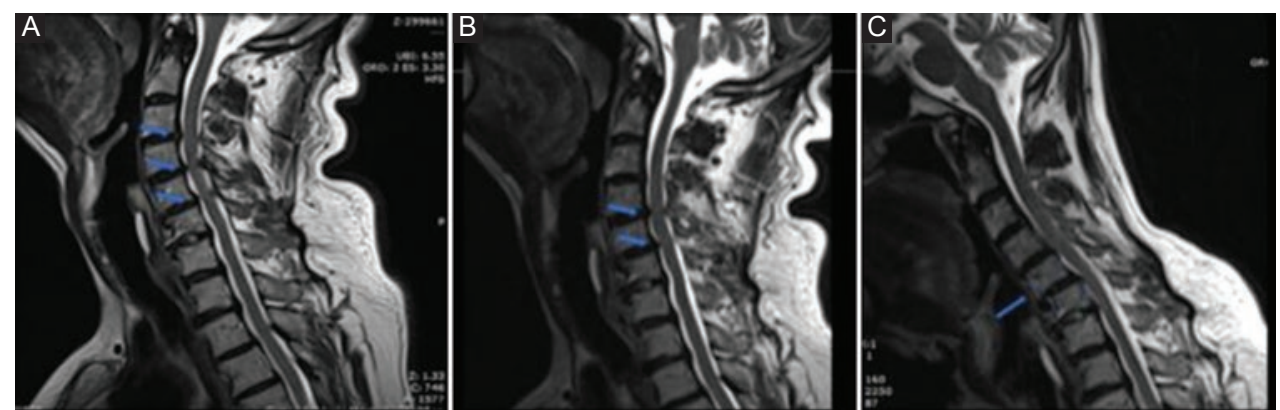

Figura 2. Varón de 64 años. A: resonancia magnética estática con protrusión discal en C3-C4, C4-C5 y C5-C6 (flechas). B: hiperextensión a $-10^{\circ}$ que muestra acentuación de las hernias discales con contacto con el cordón medular solo en dos niveles. C: flexión a $40^{\circ}$ que demuestra la inestabilidad de los cuerpos vertebrales en C4-C5 y C5-C6, que disminuyen el diámetro del canal cervical.
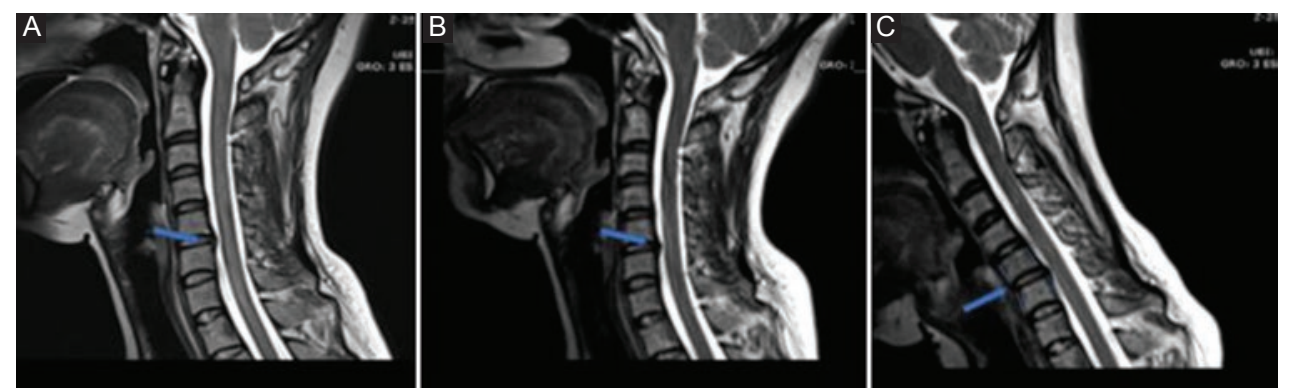

Figura 3. Mujer de 35 años. A: resonancia magnética estática con protrusión discal en C5-C6. B: hiperextensión a -10 que hace más evidente la hernia discal. C: la flexión a $40^{\circ}$ se asocia con desplazamiento de C5-C6 y disminución del espacio subdural (flecha).

de las fibras de colágeno y reducción de los proteoglicanos, con una pérdida de los componentes de agua y elasticidad del disco. Después de los 40 años de edad, el disco se vuelve más fibroso y desorganizado, lo que en un estadio final va a condicionar su colapso. Esto, a su vez, va a ser el factor principal para que se empiecen a desarrollar osteofitos, engrosamiento del ligamento amarillo y abombamiento del ligamento longitudinal posterior con estrechamiento del canal espinal, así como abombamientos del segmento posterior del disco intervertebral con afectación de los forámenes de conjunción. Por ello, la degeneración discal va a permitir un movimiento hacia delante y atrás del cuerpo vertebral adyacente, permitiendo una laxitud anómala de los ligamentos que mantienen la firmeza entre cada uno de los cuerpos vertebrales, con luxación de las facetas. Este movimiento continuo va a favorecer la formación de osteofitos y de osteoartritis, con disminución en los agujeros de conjunción y el agujero vertebral, que permitirán una coalición del ápice superior de las facetas con la pars articularis y el pedículo.

Los cambios degenerativos de las facetas articulares se presentan como un adelgazamiento del cartílago, cambios escleróticos en el hueso subcondral, formación de osteofitos, inflamación sinovial y laxitud 
ligamentaria; todos estos cambios en conjunto favorecen una hipermovilidad de la vértebra, condicionando una fractura de la pars articularis como proceso final.

La flexión y la extensión son movimientos que permiten demostrar alteraciones en la situación del disco, mismos que estarían evaluando los cambios anteriormente descritos, hallazgos que en una RMe solo evaluarían la morfología del disco, sin posibilidad de evaluar la laxitud ligamentaria y la alteración en la fibras de colágeno que mantengan el disco pulposo estable, así como la evaluación de los cambios degenerativos en las facetas articulares.

Actualmente, en México, y más en nuestra ciudad, no existen métodos que permitan la evaluación dinámica de la columna cervical con RM, por lo que la frustración y la falta de correlación clínico-radiológica es un tema importante para neurocirujanos, neurólogos, traumatólogos, etc., al no poder aportar mayor información en los pacientes que persisten con síntomas sin mejoría ante los tratamientos.

El objetivo es diseñar un dispositivo que permita evaluar los movimientos dinámicos de la columna cervical y demostrar las modificaciones que existen en las diferentes angulaciones en comparación con la RMe.

A pesar de que la muestra de pacientes es pequeña, creemos necesario continuar con la evaluación de la columna con métodos dinámicos utilizando la RM, ya que, como hemos visto, ayudó a evaluar los discos vertebrales de una manera diferente a la que hemos estado acostumbrados, determinando una inestabilidad de un $58 \%$ de los segmentos evaluados, obteniendo un riesgo relativo de 16 y un valor de $p \leq 0.05$, haciendo el estudio una prueba significativa para la evaluación de la inestabilidad de los discos vertebrales y mostrando que la RMd va a favorecer una evaluación más completa de dicha inestabilidad, 16 veces mayor que con la RM convencional. Este hallazgo es sumamente importante, ya que nos ayuda a establecer el grado de enfermedad degenerativa que tiene el paciente y a qué otros hallazgos se puede asociar, hablando de los cambios de situación agregada.

Consideramos que este método se podrá implementar a cualquier tipo de resonador siempre y cuando tenga un túnel amplio para la realización de los movimientos en flexión y extensión con el uso de dispositivos que sean compatibles con el campo magnético, para poder demostrar los cambios que puedan llegar a sugerir una inestabilidad, y esto permita demostrar la causa de la cervicalgia y que pueda otorgarse un tratamiento adecuado.

\section{Conflicto de intereses}

Los autores declaran no tener ningún conflicto de intereses respecto a este artículo.

\section{Responsabilidades éticas}

Protección de personas y animales. Los autores declaran que para esta investigación no se han realizado experimentos en seres humanos ni en animales.

Confidencialidad de los datos. Los autores declaran que han seguido los protocolos de su centro de trabajo sobre la publicación de datos de pacientes.

Derecho a la privacidad y consentimiento informado. Los autores han obtenido el consentimiento informado de los pacientes y/o sujetos referidos en el artículo. Este documento obra en poder del autor de correspondencia.

\section{Bibliografía}

1. Manchikanti L. Comprehensive review of epidemiology, scope, and impact of spinal pain. Pain Physician: guidelines special issue. 2009;12:E35-70.

2. Freund M. Degenerative spine disorders in the context of clinical findings. Eur J Radiol. 2006;58:16-26.

3. Choi K, Kim JS, Jung B, Lee SH. Dynamic lumbar spinal stenosis: the usefulness of axial loaded MRI in preoperative evaluation. J Korean Neurosurg Soc. 2009;46:265-8.

4. Birnbaum K, Uwe M. Functional cervical MRI within the scope of whiplash injuries: presentation of a new motion device for the cervical spine. Surg Radiol Anat. 2010;32:181-8.

5. Galluci M. Degenerative disease of the spine. Neuroimag Clin N Am. 2007; 17:87-103.

6. Liuke M, Soloviera S. Disc degeneration of the lumbar spine in relation to overweight. Int J Obes. 2005;29:903-8.

7. Leone A. Lumbar intervertebral instability. Radiology. 2007;245:62-7.

8. Latarjet M, Ruiz A. Anatomía humana. 4. ed. Tomo 2. Panamericana, Madrid; 2008. p. 27-59.

9. Elsig J. Dynamic imaging of the spine with an open upright MRI: present results and future perspectives of fMRI. Eur J Orthop Surg Traumatol. 2007; 17:119-24

10. Endo K, Suzuki H, Nishimura H, Tanaka H, Shishido T, Yamamoto K. Kinematic analysis of the cervical cord and cervical canal by dynamic neck motion. Asian Spine J. 2014:8:747-52.

11. Schlamann M, Reischke L, Klassen D, Maderwald S, Böhner V, Kollia K, et al. Dynamic magnetic resonance imaging of the cervical spine using the neuro Swing System. Spine (Phila Pa 1976). 2007;32:2398-401.

12. Jinkins JR, Dworkin JS, Damadian RV. Upright, weight-bearing, dynamic-kinetic MRI of the spine: initial results. Eur Radiol. 2005;41:31-5.

13. Lao $L$. Missed cervical disc bulges diagnosed with kinematic resonance imaging. Eur Spine. 2014;23:1725-9.

14. Fardon DF, Williams AL, Dohring EJ, Murtagh FR, Gabriel Rothman SL, Sze GK. Lumbar disc nomenclature: version 2.0: Recommendations of the combined task forces of the North American Spine Society, the American Society of Spine Radiology and the American Society of Neuroradiology. Spine J. 2014; 14:2525-45.

15. Bono C, Ghiselli G, Gilbert T, Kreiner DS, Reitman C, Summers JT, et al.; North American Spine Society. An evidence-based clinical guideline for the diagnosis and treatment of cervical radiculopathy from degenerative disorders. Spine J. 2011;11:64-72.

16. Henderson CM, Hennessy RG, Shuey HM Jr, Shackelford EG. Posterior-lateral foraminotomy as an exclusive operative technique for cervical radiculopathy: a review of 846 consecutively operated cases. Neurosurgery. 1983;13:504-12.

17. Hattou L, Morandi X, Le Reste PJ, Guillin R, Riffaud L, Hénaux PL. Dynamic cervical myelopathy in young adults. Eur Spine J. 2014; 23:1515-22

18. Shellock F, Powers C. Kinematic MRI of the joints, functional anatomy, kinesiology and clinical applications. Radiology. 2003;178:61-81. 